Manuelle Medizin 2016 $54: 203-204$

DOI 10.1007/s00337-016-0164-1

Online publiziert: 15 . Juli 2016

(c) Springer-Verlag Berlin Heidelberg 2016

CrossMark

\section{S. Kopp}

Poliklinik für Kieferorthopädie, Zentrum der Zahn-, Mund- und Kieferheilkunde "Carolinum", GoetheUniversität Frankfurt am Main, Frankfurt am Main, Deutschland

\title{
Mutig die Schätze manuellen Könnens wissenschaftlich korrekt heben
}

Liebe Leserinnen, lieber Leser!

\section{Seien Sie mutig!}

Wir haben gerade am Wochenende einen interdisziplinären Kurs zum Anfassen und Bewegen im eigentlichen Sinne in der Zahnärztekammer Hessen in Frankfurt im erstklassigen kollegialen Miteinander erleben dürfen. Da hatte die MWE doch tatsächlich den Mut gehabt, in Frankfurt einen 3-Tage-Kurs zum Thema „Kiefergelenk - eine interdisziplinäre Aufgabe für die Zahnmedizin und die manuelle Medizin" anzubieten. Und die Zahnärztekammer lieferte dazu das perfekte organisatorische Umfeld für die Veranstaltung. Der Kurs war so voll, dass gerade noch ein vernünftiges gemeinsames Arbeiten möglich war. Das war noch nichts Außergewöhnliches. Aber nach den ersten Minuten kamen Diskussionen auf, die wir uns als Kursleiter zwar gewünscht, aber nicht erwartet haben. Es folgten 3 Tage des kollegialen - interdisziplinären - Miteinanders. Schon wenige Tage danach haben sich erstaunlich viele Kolleg(inn)en gemeldet und davon berichtet, dass es einerseits sehr schwer sei, sich als „Manuali“ mit den Zahnärzten und Kieferorthopäden vor Ort abzustimmen, es aber andererseits auch völlig interessant und befriedigend sei, dass man im ersten noch vorsichtigen Miteinander bisher kaum Denkbares erreichen konnte.

\section{Wovor schrecken wir zurück?}

Ist es die Bequemlichkeit, die uns daran hindert, auf Kolleg(inn)en zuzugehen und ggf. in fremder Praxis gemeinsam zu behandeln? Ist es unsere immer präsente Überarbeitung und der dazu gehörende Zeitstress und das schlechte Gewissen, schon wieder die Familie zu vernachlässigen? Ist es die Literaturgläubigkeit - und die Literatur ist wahrhaft spärlich im evidenzbasierten Sinne -, die uns glauben macht, alles, was mit Kiefergelenk zu tun hat, sei sowieso psychisch bedingt und gehört nicht in die Hände der manuell Tätigen? Ungeachtet dessen haben gerade die Beiträge in dieser Zeitschrift immer wieder belegt, dass es klinisch funktioniert, auch wenn wir keine Publikationen mit Evidenzniveau 2 liefern können. Wen wundert es denn? Gernot Plato schrieb mit mir schon 1994 in der Zeitschrift Manuelle Medizin, dass die Praktiker etwas tun müssen - ja er forderte, dass die Praktiker den Wissenschaftlern die Fragen stellen müssen, die dann mit den entsprechenden Werkzeugen in der Klinik zu beantworten sind.

\section{Was ist mit den Antworten?}

Wenn wir uns fragen, was nach 30 Jahren interdisziplinärer Arbeit geblieben ist, schlägt eine täglich größer werdende Empirie zu Buche, aber die Evidenz fehlt noch viel zu oft. Eines ist sicher: Die chirurgische Orientierung der vermeintlich neuen Orthopädie trägt nichts, aber auch gar nichts zur Lösung der vielen meist durch kollegiale Unkenntnis und kollegiale Mutlosigkeit schon chronisch gewordenen Probleme der „Kiefergelenkpatienten" bei. Dieser verbale Wutschrei eines Wissenschaftlers, der gleichzeitig überzeugter Kliniker ist, gilt sowohl für die Zahnheilkunde und Kieferorthopädie als auch für die manuelle Medizin.

\section{Was wünscht sich ein Autor in einer kiefergelenklastigen Ausgabe der Manuellen Medizin?}

Ich wünsche mir Kolleginnen und Kollegen, wie ich sie am Wochenende erlebt habe. Starker Tobak, das alles zu verinnerlichen, was da theoretisch und manuell nachvollziehbar mitzunehmen als Angebot im Raume stand. Gratulation der Sache, dass so viele Mutige dabei waren, anzufangen. Wir haben am Samstag vereinbart, dass wir uns gegenseitig auf dem Laufenden halten. Natürlich brauchen wir in der Klinik Sie persönlich als Vielbehandler in der Praxis. Die Zahlen, die Sie haben, können wir bei der schier unendlichen Zahl der überkapazitär und damit ,übercurricular"Studierenden, die wir zur klinischen Sicherheit führen, niemals erreichen. Allerdings hat die momentane Situation, nämlich dass sehr viele junge hochmotivierte Kollegen aufdem Weg zu Approbation sind, auch eine Reihe von interessanten und faszinierenden Effekten. Viele wollen promovieren. Wir haben die Themen in Überfülle. Nur die Personalknappheit schränkt unsere Möglichkeiten ein. Lamentieren ist schon lange nicht mehr das Werkzeug einer ausbildenden Universitätsmedizin, die von hochgelobten und superfinanzierten Exzellenzclustern in der Lehre und der möglichen „Restforschung“ überrollt wird. Wir krempeln die Ärmel hoch und arbeiten mit den Jungen. Fleißig, sicher, zielstrebig, kollegial und streng geht es 
da zu, oft 7 Tage in der Woche. Aber es bringt Freude und Zufriedenheit. Viele von Ihnen haben - wie Plato schon 1994 in unserer Zeitschrift geschrieben hat die Möglichkeiten.

Haben Sie einfach den Mut, Ihre Universität im Allgemeinen und natürlich auch mich im Speziellen anzusprechen, ob man denn aus der Dokumentation Ihrer vielen Fälle und mit Unterstützung unserer zukünftigen Generation dank Ihrer Erfahrung nicht doch - auch wenn es „nur“'retrospektiv ist - die Schätze manuellen Könnens wissenschaftlich korrekt heben kann.

Ich schreibe dies auch, weil ich Lothar Beyer explizit in dem Vorhaben unterstützen möchte, dass unsere Zeitschrift im Web of Science aufgenommen wird. Wir diskutieren das Thema schon lange - ohne greifbares Ergebnis.

\section{Was ist los mit uns?}

Mir scheint, wir haben bisher den Kopf in den Sand gesteckt, weil wir erstens Angst vor unseren eigenen Ergebnissen und der Notwenigkeit lückenloser Dokumentation haben und zweitens vor der Evidenzforderung aus der Sicht der Praxis sowieso in die Knie gehen. Wenn es uns wirklich ernst ist mit der manuellen Medizin inklusive Osteopathie, sollten wir uns endlich darum kümmern, dass wir die jungen Leute motivieren, in unserer Zeitschrift über die uns wichtigen Dinge zu publizieren. Ich zähle mich gegenwärtig zu einem zwar jung gebliebenen, aber real in die Jahre gekommenen „Altenclub" der manuellen Medizin. Wie können wir das ändern, auch wenn z. B. ich selbst aus derzeitiger Sicht immerhin noch 12 Jahre den Lehrstuhl für unsere Überzeugung nutzen kann? Wenn wir ehrlich sind, bringen wir nur "gute“ Arbeiten in die Manuelle Medizin, wenn wir diese streng dem Peer-Review-Verfahren unterziehen lassen und uns mit nicht $\mathrm{zu}$ unterschätzender Unterstützung des Verlags die Pflicht auferlegen, in möglichst naher Zukunft unsere Zeitschrift im Web of Science aufnehmen zu lassen.

Bitte machen Sie mit! Unser Fach und unsere gemeinsame Überzeugung haben Ihr Engagement verdient. Die Zeit, hinter dem Ofen zu warten und nichts zu unter- nehmen, wird kurz und kürzer. Ich schlage vor, dass wir das Zepter in die Hand nehmen und uns zur Aktivität für die Manuelle Medizin zwingen - ein Zwang, der hoffentlich für viele von uns zur Freude wird. Lothar Beyer und ich, aber nicht nur wir allein, zählen auf Ihre Unterstützung durch Ihre aktive Mithilfe.

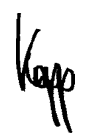

\section{S. Kopp}

\section{Korrespondenzadresse}

\section{Prof. Dr. S. Kopp}

Poliklinik für Kieferorthopädie, Zentrum der Zahn-, Mund- und Kieferheilkunde "Carolinum", Goethe-Universität Frankfurt am Main Theodor-Stern-Kai 7, Haus 29, 60590 Frankfurt am Main, Deutschland

kopp@med.uni-frankfurt.de

Interessenkonflikt. S. Kopp gibt an, dass kein Interessenkonflikt besteht.

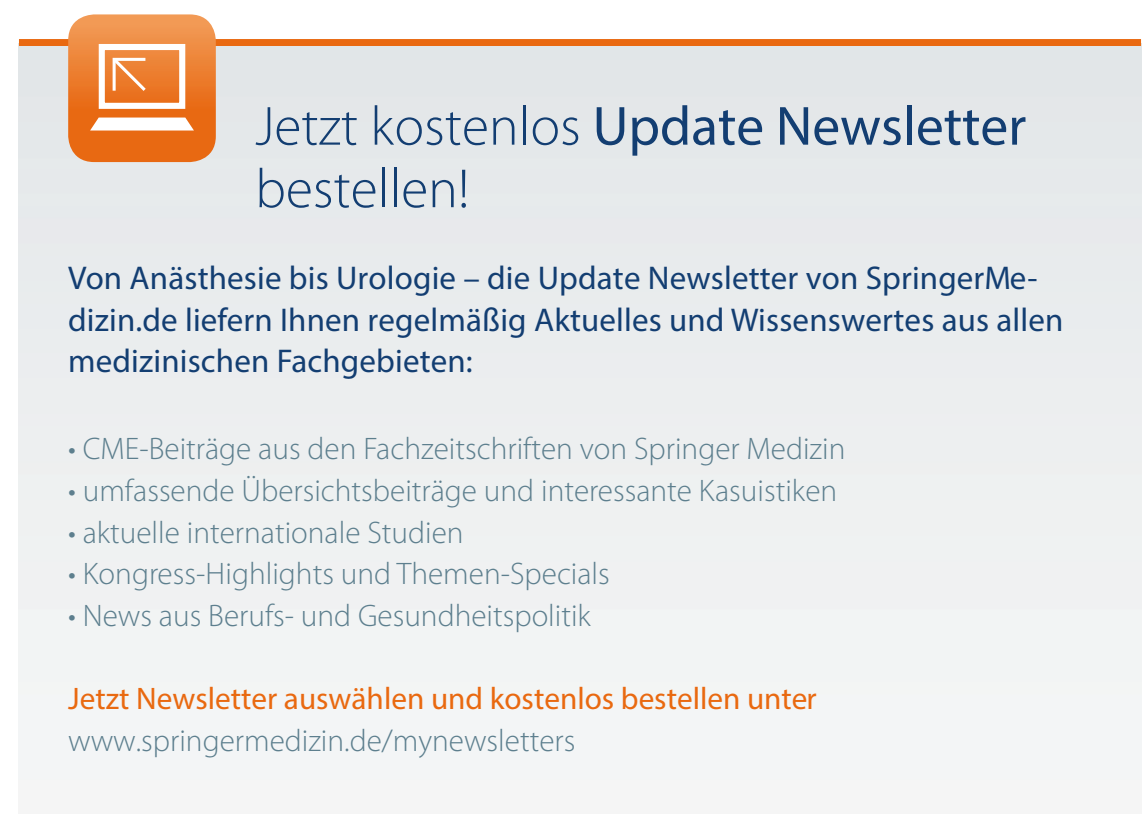

